\title{
Real-World Tumor Response of Palbociclib Plus Letrozole Versus Letrozole for Metastatic Breast Cancer in US Clinical Practice
}

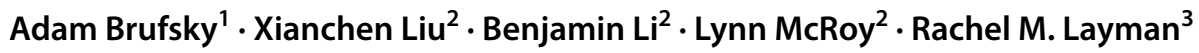

Accepted: 21 June 2021

(c) The Author(s) 2021, corrected publication 2021

\begin{abstract}
Background Limited information exists regarding tumor response to palbociclib plus an aromatase inhibitor (AI) versus AI alone in real-world practice.

Objective To evaluate the real-world tumor response of palbociclib plus letrozole (PAL+LET) versus LET alone as first-line treatment for patients with hormone receptor-positive/human epidermal growth factor receptor 2-negative metastatic breast cancer (HR+/HER2- MBC) in routine US clinical practice.

Patients and Methods This retrospective analysis included HR+/HER2- MBC patients who initiated PAL+LET or LET as first-line treatment between February 2015 and September 2018 in the Flatiron Health Analytics database. Patients were followed until December 2018. Real-world best tumor response (rwBTR) was determined based on physicians' assessment of radiologic evidence for change in burden of disease.

Results Of the 1383 eligible patients who initiated PAL+LET or LET as first-line therapy in the Flatiron database, 968 patients had $\geq 1$ tumor response assessment (662 received PAL+LET and 306 received LET). The rwBTR rate (complete response+partial response) in the first-line setting was 59.8\% in the PAL+LET group and 39.2\% in the LET group (odds ratio 2.31 (95\% CI 1.75-3.04), $P<0.0001$ ). After $1: 1$ propensity-score matching, the rwBTR rate was $58.6 \%$ in the PAL+LET group versus $39.1 \%$ in the LET group (odds ratio 2.21 (95\% CI 1.50-3.25), $P<0.0001$ ).

Conclusions This real-world analysis demonstrated that HR+/HER2- MBC patients were more likely to respond to PAL+LET compared to LET. These findings further showed the effectiveness of PAL+LET therapy in the real-world setting and support the combination as a standard of care for MBC.
\end{abstract}

Study Registration Pfizer; NCT04176354; registered November 25, 2019

Digital Features for this article can be found at https://doi.org/ 10.6084/m9.figshare.14931843.

Adam Brufsky

brufskyam@upmc.edu

$\triangle$ Xianchen Liu

Jasonxc.Liu@pfizer.com

1 College of Medicine, University of Pittsburgh, 300 Halket St., Suite 4628, Pittsburgh, PA 15213, USA

2 Pfizer Inc, New York, NY, USA

3 The University of Texas MD Anderson Cancer Center, Houston, TX, USA

\section{Key Points}

This is the first comparative analysis of real-world tumor response in patients with hormone receptor-positive/ human epidermal growth factor receptor 2-negative metastatic breast cancer (HR+/HER2- MBC) treated with first-line palbociclib plus letrozole versus letrozole alone in the real-world clinical setting.

Patients with HR+/HER2- MBC were more likely to respond to palbociclib plus letrozole compared with letrozole alone.

These data complement the clinical benefit of palbociclib plus endocrine therapy observed in randomized clinical trials and support palbociclib plus letrozole as a standard of care for patients with HR+/HER2- MBC. 


\section{Plain Language Summary}

Palbociclib (Ibrance $®$; PAL) is a treatment for advanced or metastatic breast cancer (MBC for short). Metastatic means that the cancer has spread from breast tissue to other areas of the body. PAL is taken with medications known as hormone therapy, such as letrozole (LET), to treat people with a type of MBC called hormone receptor-positive, human epidermal growth factor receptor 2-negative (HR+/HER2-). Researchers wanted to understand more about how treatment with PAL+LET affected tumor response (i.e., if the tumor shrinks or disappears) in women with HR+/HER2- MBC. We looked at tumor response to PAL+LET in a real-life setting using anonymous medical record information from a database of about 2.4 million US patients with cancer. This study included 1383 patients with HR+/HER2- MBC who started PAL+LET (754 patients) or LET alone (629 patients) as their first MBC treatment from 2015-2018. Among people who took PAL+LET, 60\% people had a tumor response compared with $39 \%$ of people who took LET alone. Of the people who took PAL+LET, half of the people lived with their cancer without it getting worse for at least 20 months compared with 15 months for people who took LET alone. These results add to what is known about PAL+LET treatment based on routine clinical practice and clinical trials. Both types of information provide support for PAL+LET treatment as the standard care for women with HR+/HER2-MBC.

\section{Introduction}

In the USA, it was estimated that 276,480 women would be diagnosed with invasive breast cancer and 42,170 women would die from breast cancer in 2020 [1]. The majority of patients with breast cancer have hormone receptor-positive/ human epidermal growth factor receptor 2-negative (HR+/ HER2-) disease [2], and approximately one-third of those diagnosed with early breast cancer eventually develop metastatic disease, for which there is no cure [3,4]. A cyclindependent kinase 4/6 (CDK4/6) inhibitor, such as palbociclib, in combination with endocrine therapy (ET) has been recommended as standard of care for patients with $\mathrm{HR}+/$ HER2 - advanced or metastatic breast cancer (MBC) [5, 6].

In the PALOMA-2 clinical study, first-line palbociclib plus letrozole therapy significantly improved median progression-free survival (PFS) compared with placebo plus letrozole (27.6 vs. 14.5 months; $P<0.0001$; data cutoff: 31 May 2017) in postmenopausal women with estrogen receptor-positive (ER+)/HER2- MBC [7]. The objective response rate (defined as confirmed complete response (CR) or partial response (PR) based on Response Evaluation Criteria in Solid Tumors (RECIST)) was $42.1 \%$ with palbociclib plus letrozole compared with $34.7 \%$ with placebo plus letrozole (odds ratio (OR), 1.40 (95\% confidence interval (CI), 0.98-2.01), $P=0.06$ ) [8].

Using real-world evidence to complement data from randomized controlled clinical studies is important because the stringent inclusion and exclusion criteria of oncology clinical studies exclude a substantial proportion of the patient population with cancer $[9,10]$. The evaluation of multiple clinical outcomes is also important to further understand the effectiveness of treatments in the real-world setting. The various realworld endpoints have unique strengths and limitations [11, 12]. Real-world time to treatment failure is defined as the time from the start of treatment to treatment discontinuation for any reason, including death, disease progression, or adverse events [13]. However, time to treatment failure is usually not recommended as an outcome for the regulatory approval evaluation of a new treatment because many factors may affect the time to treatment failure [13]. Real-world time to next treatment, which is defined as the time from the end of the initial treatment to the start of the next treatment, is another clinical outcome that may be evaluated, but is limited by variability in practice patterns and inaccuracy in capturing precise times of treatment initiation [12]. Real-world tumor response is evaluated based on radiologic evidence for change in disease burden throughout the course of treatment as assessed by the treating physician [14]. Real-world PFS (rwPFS) is defined as the time from the start of treatment to death or disease progression, with the treating clinician determining disease progression using radiology, pathology, laboratory findings, or clinical assessment [14]. Real-world tumor response may be assessed more reliably and objectively than rwPFS; however, there is a lack of uniformity in the timing or frequency and criteria of tumor assessment during treatment in clinical practice [12]. In addition, real-world tumor response alone may under-estimate treatment effectiveness as many patients derive benefit from prolonged stable disease [12]. Therefore, evaluation of the clinical effectiveness of a treatment utilizing multiple outcome measures is more robust and can provide a more complete understanding of treatment benefit than using any one real-world endpoint.

Findings from small and single-arm real-world studies evaluating rwPFS and OS outcomes have supported the benefit of palbociclib observed in clinical trials [15-21]. A realworld comparative effectiveness analysis using the Flatiron Health Analytics database showed that first-line palbociclib plus letrozole was more effective than letrozole alone based on median real-world PFS (20.2 vs. 11.9 months; hazard ratio, 0.54 (95\% CI 0.46-0.65); $P<0.0001)$ and overall survival (OS) results (not reached vs. 43.1 months; hazard ratio 0.58 (95\% CI 0.46-0.73); $P<0.0001)$ after propensity-score matching (PSM) [22]. Additionally, a recent real-world study using the Flatiron Health database evaluated tumor response to abemaciclib in 118 patients with HR+/HER2- MBC who 
received abemaciclib across various lines of treatment [23]. The analysis showed that $41.2 \%$ of patients had a real-world best response (defined as real-world CR or PR).

There is limited information, however, regarding tumor response to palbociclib plus an aromatase inhibitor (AI) compared with an AI alone in real-world clinical practice [24]. A multi-country retrospective medical chart review of patients with HR+/HER2 - advanced breast cancer (ABC) or MBC, who received palbociclib in combination with either an AI or fulvestrant in real-world clinical practice, reported a real-world objective response rate (based on physicians' assessments) of $79.5 \%$ in the USA and $66 \%$ in Argentina [19]. Additionally, a small real-world study of patients with HR+/HER2- ABC or MBC treated with palbociclib plus an AI or fulvestrant in Italy demonstrated a real-world objective response rate of $31 \%$ [21]. The variation in response rates observed could be attributed to differences in patient characteristics as well as the methods and criteria used to assess tumor response.

To add to the rwPFS and OS results previously reported from the Flatiron Health Analytics database [22], this retrospective analysis (using the same database) describes demographic and clinical characteristics and real-world best tumor response (rwBTR, defined as CR or PR based on the treating clinician's assessment of radiologic evidence) among a large sample of HR+/HER2- MBC patients initiating palbociclib plus letrozole compared with letrozole alone as first-line therapy in routine US clinical practice. This analysis also evaluated rwBTR rates by subgroup, including by visceral disease and bone-only metastases.

\section{Methods}

\subsection{Study Design and Data Source}

This retrospective analysis of electronic health records (EHRs) was performed using de-identified patient data from the Flatiron Health Analytics database. The Flatiron longitudinal database comprises structured and unstructured EHRs from $>280$ cancer clinics and $>800$ sites of care, representing approximately 2.4 million US patients with cancer who are actively being treated for their disease. Previous realworld cancer studies have demonstrated that this database has appropriate characteristics to assess treatment patterns and clinical outcomes $[14,25,26]$.

\subsection{Patients}

Women aged $\geq 18$ years with documented HR+/HER2MBC were included in this analysis. Patients had a date of first prescription (index date) for palbociclib plus letrozole or letrozole alone as first-line therapy for MBC between 3 February 2015, and 30 September 2018, and potential follow-up for $\geq 3$ months from the index date to the study cutoff date of 31 December 2018. Patients were excluded if they had prior treatment with CDK4/6 inhibitors, AIs, fulvestrant, raloxifene, tamoxifen, or toremifene in the metastatic setting; had a first structured activity $>90$ days after MBC diagnostic date; or received treatment with a CDK4/6 inhibitor as part of a clinical trial.

\subsection{Outcomes}

The rwBTR $(\mathrm{CR}+\mathrm{PR})$, which occurred $\geq 30$ days after therapy initiation, was assessed during first-line therapy using the treating clinician's assessment of radiologic evidence for change in burden of disease over the course of the treatment [14]. Unstructured physician notes and radiology reports from electronic health records were reviewed retrospectively to determine rwBTR category. CR was defined as the complete resolution of all visible disease, and PR was defined as a partial reduction in size of visible disease in some or all areas without any areas of increase in visible disease. Notably, PR should have captured a size decrease even though disease was still present. Patients with stable disease (SD) had no change in the overall size of visible disease; SD was also used for "mixed response," when patients had some lesion size increase and some lesion size decrease. Progressive disease was defined as an increase in visible disease and/or the presence of any new lesions. In situations in which the clinician explicitly stated that he or she could not make a determination of the assessment, response was classified as indeterminate.

Real-world PFS was defined as the time from the start of treatment with palbociclib plus letrozole or letrozole alone to death or disease progression. Disease progression was concluded by the treating clinician based on radiology, pathology, laboratory evidence, or clinical assessment [14]. Patients who did not die or have disease progression were censored at the date of initiation of the next line of therapy for those with two or more lines of therapy or their last visit during the study period (February 2015-December 2018) for patients with only one line of therapy.

Overall survival (OS) was defined as the time from the start of treatment with palbociclib plus letrozole or letrozole alone to death from any cause recorded by Flatiron in the data extract. The date of death was obtained from a recent mortality data set generated by combining multiple data sources and benchmarked against the National Death Index [25], which is widely considered a gold standard mortality data set in the USA [27]. Patients who did not die were censored at the time of study cutoff (31 December 2018). 


\subsection{Statistical Analyses}

Patient characteristics and real-world best tumor response were shown using descriptive statistics. A multivariable binomial logistic regression model was used to generate propensity scores (PS) [28]. Variables used for computing PS are presented in Table 1. Individual patients in one cohort were matched 1:1 to patients in the other cohort based on closest PS using a caliper of 0.01 [28]. Logistic regression was used to estimate the odds of real-world best tumor response in the palbociclib plus letrozole group compared with the letrozole alone group. Survival time was calculated using Kaplan-Meier survival analyses. To compare the risk of rwPFS and OS between the matched study cohorts, Cox proportional hazards models with a robust sandwich estimator were used. As a sensitivity analysis, a stabilized inverse probability of treatment weighting analysis was performed.

\section{Results}

\subsection{Study Patients}

Between 3 February 2015, and 30 September 2018, 1,383 women with HR+/HER2- MBC initiated palbociclib plus letrozole $(n=754)$ or letrozole alone $(n=629)$ as first-line therapy in the Flatiron database. Among these patients, 662 patients received palbociclib plus letrozole and 306 patients received letrozole alone and had one or more tumor response assessments during first-line therapy. A total of 430 patients with one or more tumor response assessments were 1:1 PSmatched, including 215 patients who received palbociclib plus letrozole and 215 patients who received letrozole alone. After stabilized inverse probability of treatment weighting, the number of patients increased to 715 patients in the palbociclib plus letrozole group and 547 patients in the letrozole alone group.

The median duration of follow-up was 20.6 months in the palbociclib plus letrozole group and 22.3 months in the letrozole alone group. Among PS-matched patients, the median duration of follow-up was 20.2 months with palbociclib plus letrozole and 23.4 months with letrozole alone. In the stabilized inverse probability of treatment weighting cohort, the median duration of follow-up was 20.5 months in the palbociclib plus letrozole group and 20.4 in the letrozole alone group.

In the unadjusted cohort, patient demographic and clinical characteristics were different between the palbociclib plus letrozole and letrozole alone groups (Table 1). After PSM, patient characteristics were generally balanced between the matched cohorts (Table 1). After stabilized inverse probability of treatment weighting, patient characteristics were generally similar between the treatment groups (Supplemental Material, Online Resource 1).

\subsection{Real-World Best Tumor Response}

In the unadjusted cohort, the rwBTR rate $(\mathrm{CR}+\mathrm{PR})$ in the first-line setting was $59.8 \%$ in the palbociclib plus letrozole group and $39.2 \%$ in the letrozole alone group (OR, 2.31 (95\% CI 1.75-3.04), $P<0.0001$; Fig. 1). After PSM, the rwBTR rate was $58.6 \%$ with palbociclib plus letrozole compared with $39.1 \%$ with letrozole alone (OR, 2.21 (95\% CI $1.50-3.25), P<0.0001)$. Similarly, stabilized inverse probability of treatment weighting analysis showed a rwBTR rate of $59.3 \%$ in the palbociclib plus letrozole group and $41.5 \%$ in the letrozole alone group (OR, 2.05 (95\% CI 1.64-2.57), $P<0.0001)$. An analysis of rwBTR rates by subgroups, including by visceral disease, generally showed a consistent benefit of palbociclib plus letrozole versus letrozole alone; no significant interactions were demonstrated in any of the subgroups analyzed (Fig. 2).

\subsection{Real-World Progression-Free Survival and Overall Survival}

Among patients with one or more tumor response assessments, treatment with palbociclib plus letrozole was associated with significantly longer $(P<0.001)$ median rwPFS versus letrozole alone in the unadjusted analysis, after PSM, and after stabilized inverse probability of treatment weighting (Fig. 3). In the unadjusted cohort, median rwPFS was 20.0 (95\% CI 17.3-23.3) months with palbociclib plus letrozole and 15.1 (95\% CI 12.1-18.5) months with letrozole alone (hazard ratio 0.70 (95\% CI 0.58-0.84), $P=0.0002$ ). After PSM, median rwPFS was 27.4 (95\% CI 20.5-31.3) months in the palbociclib plus letrozole group and 15.4 (95\% CI 12.1-18.5) months in the letrozole alone group (hazard ratio, 0.60 (95\% CI 0.46-0.79), $P=0.0002$ ). In the stabilized inverse probability of treatment weighting analysis, median rwPFS was 20.2 (95\% CI 17.7-24.4) months in the palbociclib plus letrozole group and 16.9 (95\% CI 12.5-21.0) months in the letrozole alone group (hazard ratio, 0.71 (95\% CI 0.60-0.83), $P<0.0001$ ).

Median OS was 43.4 (95\% CI 33.5-not estimable [NE]) months with letrozole alone and not reached with palbociclib plus letrozole in the unadjusted analysis (hazard ratio 0.61 (95\% CI 0.48-0.79), $P=0.0001$; Fig. 4). After PSM, median OS was not reached in either the palbociclib plus letrozole or letrozole alone group (hazard ratio 0.58 (95\% CI 0.39-0.85), $P=0.0052$ ). In the stabilized inverse probability of treatment weighting analysis, median OS was not reached with palbociclib plus letrozole and was 43.5 (95\% CI 33.5-NE) months with letrozole alone (hazard ratio 0.65 (95\% CI 0.53-0.81), $P<0.0001)$. 
Table 1 Patient characteristics

\begin{tabular}{|c|c|c|c|c|c|c|}
\hline \multirow[t]{2}{*}{ Characteristic } & \multicolumn{3}{|l|}{ Unadjusted cohort } & \multicolumn{3}{|l|}{ Cohort after PSM } \\
\hline & $\begin{array}{l}\text { Palbociclib } \\
\text { + Letrozole } \\
(n=662)\end{array}$ & Letrozole $(n=306)$ & $\begin{array}{l}\text { Standardized } \\
\text { difference }\end{array}$ & $\begin{array}{l}\text { Palbociclib } \\
+ \text { Letrozole } \\
(n=215)\end{array}$ & Letrozole $(n=215)$ & $\begin{array}{l}\text { Standardized } \\
\text { difference }\end{array}$ \\
\hline \multicolumn{7}{|l|}{ Age, y } \\
\hline Mean (SD) & $64.4(11.2)$ & $70.6(10.9)$ & 0.5645 & $68.3(10.8)$ & $68.0(10.8)$ & -0.0288 \\
\hline Median (IQR) & $65.0(57.0-73.0)$ & $72.0(63.0-80.0)$ & & $70.0(62.0-76.0)$ & $68.0(61.0-78.0)$ & \\
\hline \multicolumn{7}{|l|}{ Age group, $n(\%), \mathrm{y}$} \\
\hline $18-64$ & $318(48.0)$ & $85(27.8)$ & -0.4270 & 68 (31.6) & $77(35.8)$ & 0.0886 \\
\hline $65-74$ & $220(33.2)$ & $90(29.4)$ & -0.0825 & $81(37.7)$ & $76(35.4)$ & -0.0483 \\
\hline$\geq 75$ & $124(18.7)$ & $131(42.8)$ & 0.5404 & $66(30.7)$ & $62(28.8)$ & -0.0407 \\
\hline \multicolumn{7}{|l|}{ Race/ethnicity, $n(\%)$} \\
\hline White & $462(69.8)$ & $216(70.6)$ & 0.0175 & $162(75.4)$ & $147(68.4)$ & -0.1556 \\
\hline Black & $46(7.0)$ & $22(7.2)$ & 0.0094 & $10(4.7)$ & $15(7.0)$ & 0.0995 \\
\hline Asian & $15(2.3)$ & $6(2.0)$ & -0.0212 & $3(1.4)$ & $5(2.3)$ & 0.0689 \\
\hline Hispanic or Latino & $16(2.4)$ & $6(2.0)$ & -0.0312 & $4(1.9)$ & $4(1.9)$ & 0.0000 \\
\hline Other/unknown & $123(18.6)$ & $56(18.3)$ & -0.0072 & $36(16.7)$ & $44(20.5)$ & 0.0957 \\
\hline \multicolumn{7}{|l|}{ Practice type, $n(\%)$} \\
\hline Academic & $36(5.4)$ & $15(4.9)$ & 0.0242 & $17(7.9)$ & $12(5.6)$ & 0.0928 \\
\hline Community & $626(94.6)$ & $291(95.1)$ & & $198(92.1)$ & $203(94.4)$ & \\
\hline \multicolumn{7}{|c|}{ Disease stage at initial diagnosis, $n(\%)$} \\
\hline I or II & $239(36.1)$ & $106(34.6)$ & -0.0306 & $65(30.2)$ & $72(33.5)$ & 0.0699 \\
\hline III & $92(13.9)$ & $45(14.7)$ & 0.0231 & $20(9.3)$ & $32(14.9)$ & 0.1718 \\
\hline IV & $274(41.4)$ & $118(38.6)$ & -0.0577 & $107(49.8)$ & $84(39.1)$ & -0.2166 \\
\hline Not documented & $57(8.6)$ & $37(12.1)$ & 0.1145 & $23(10.7)$ & $27(12.6)$ & 0.0581 \\
\hline \multicolumn{7}{|c|}{ ECOG performance status, $n(\%)$} \\
\hline 0 & $278(42.0)$ & $72(23.5)$ & -0.4013 & $62(28.8)$ & $62(28.8)$ & 0.0000 \\
\hline 1 & $138(20.9)$ & $60(19.6)$ & -0.0308 & $43(20.0)$ & $47(21.9)$ & 0.0457 \\
\hline 2 & $36(5.4)$ & $22(7.2)$ & 0.0721 & $17(7.9)$ & $16(7.4)$ & -0.0175 \\
\hline 3 or 4 & $4(0.6)$ & $11(3.6)$ & 0.2097 & $3(1.4)$ & $2(0.9)$ & -0.0434 \\
\hline Not documented & $206(31.1)$ & $141(46.1)$ & 0.3110 & $90(41.9)$ & $88(40.9)$ & -0.0189 \\
\hline \multicolumn{7}{|l|}{ Visceral disease, ${ }^{\mathrm{a}} n(\%)$} \\
\hline No & $378(57.1)$ & $210(68.6)$ & -0.2403 & $137(63.7)$ & $138(64.2)$ & -0.0097 \\
\hline Yes & $284(42.9)$ & $96(31.4)$ & & $78(36.3)$ & $77(35.8)$ & \\
\hline Bone-only disease, ${ }^{\mathrm{b}} n(\%)$ & $246(37.2)$ & $121(39.5)$ & 0.0490 & $96(44.7)$ & $89(41.4)$ & -0.0658 \\
\hline Brain metastases, $n(\%)$ & $16(2.4)$ & $14(4.6)$ & 0.1177 & $4(1.9)$ & $7(3.3)$ & 0.0885 \\
\hline \multicolumn{7}{|c|}{ Number of metastatic sites, ${ }^{c} n(\%)$} \\
\hline 1 & $326(49.2)$ & $172(56.2)$ & 0.1398 & $127(59.1)$ & $123(57.2)$ & -0.0377 \\
\hline 2 & $193(29.2)$ & $71(23.2)$ & -0.1357 & $52(24.2)$ & $50(23.3)$ & -0.0219 \\
\hline 3 & $88(13.3)$ & $33(10.8)$ & -0.0772 & $25(11.6)$ & $27(12.6)$ & 0.0285 \\
\hline$\geq 4$ & $45(6.8)$ & $9(2.9)$ & -0.1799 & $7(3.3)$ & $8(3.7)$ & 0.0254 \\
\hline Not documented & $10(1.5)$ & $21(6.9)$ & 0.2696 & $4(1.9)$ & $7(3.3)$ & 0.0885 \\
\hline
\end{tabular}

The balance in important prognostic baseline characteristics was assessed using a standardized difference approach, with a standardized difference of $\geq 0.10$ considered indicative of practical significance [28]

ECOG Eastern Cooperative Oncology Group, IQR interquartile range, PSM propensity-score matching

${ }^{a}$ Visceral disease was defined as metastatic disease in the lung and/or liver; patients could have had other sites of metastases. No visceral disease was defined as no lung or liver metastases

${ }^{\mathrm{b}}$ Bone-only disease was defined as metastatic disease in the bone only

${ }^{\mathrm{c}}$ Multiple metastases at the same site were counted as one site (e.g., if a patient had three bone metastases in the spine, it was considered only one site) 


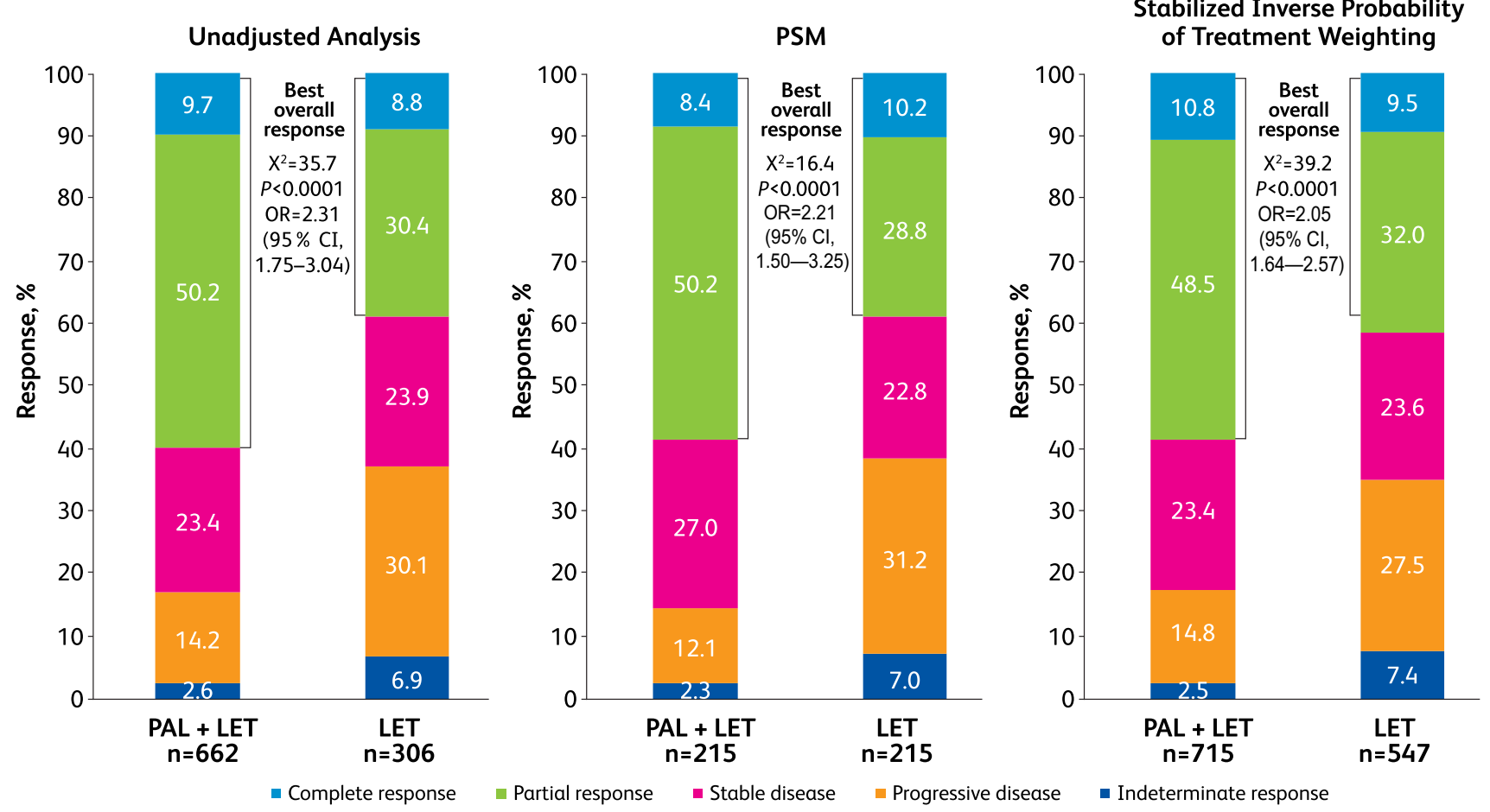

Fig. 1 Real-world best tumor response. ECOG Eastern Cooperative Oncology Group, LET letrozole, $O R$ odds ratio, $P A L$ palbociclib, $P S M$ propensity-score matching. Indeterminate response includes sit-

\section{Discussion}

To our knowledge, this is the first comparative analysis of real-world tumor response in patients with HR+/HER2MBC treated with first-line palbociclib plus letrozole versus letrozole alone in the real-world clinical setting. Tumor response assessments are important in clinical practice when treating patients with cancer [29]. A previous study has shown that partial or complete tumor response is associated with cancer-related symptom improvement in patients with MBC [30]. Moreover, early identification of nonresponding tumors and ineffective therapies are important to minimize the risk of disease progression and to decrease the extent of visceral disease [29].

Findings from this comparative analysis showed that patients treated in the first-line setting with palbociclib plus letrozole had an associated increased likelihood of tumor response compared with those treated with letrozole alone (after PSM, 58.6\% vs. 39.1\%, respectively; OR 2.21 (95\% CI 1.50-3.25), $P<0.0001)$. Additionally, a generally consistent benefit of palbociclib plus letrozole versus letrozole alone was shown across subgroups, including by visceral disease and presence of bone-only metastases, with no significant interactions demonstrated in any of the subgroups evaluated. These findings complement data from randomized clinical studies showing the efficacy of palbociclib plus letrozole [7, 8, 31]. uations in which the clinician explicitly states that he/she is not able to make a determination of the assessment

Patients included in the PALOMA-2 clinical study were postmenopausal with ER+/HER2- ABC who had not received prior systemic therapy for advanced disease [8]. Among all the randomized patients, the objective response rate was $42.1 \%$ (95\% CI 37.5-46.9) with palbociclib plus letrozole and $34.7 \%$ (95\% CI 28.4-41.3) with placebo plus letrozole [8]. Among patients who had measurable disease based on the Response Evaluation Criteria in Solid Tumors (RECIST) criteria, the objective response rate was $55.3 \%$ (95\% CI 49.9-60.7) with palbociclib plus letrozole and 44.4\% (95\% CI 36.9-52.2) with placebo plus letrozole [8]. Results from this comparative analysis also demonstrated that the real-world CR rates were similar between patients treated with palbociclib plus letrozole and those who received letrozole alone (approximately $10 \%$ in both groups before and after PSM/stabilized inverse probability of treatment weighting). These results complement the $\mathrm{CR}$ rates reported in postmenopausal women with $\mathrm{ER}+$ / HER2- ABC who received first-line palbociclib plus letrozole or letrozole alone (1\% in both groups) in the PALOMA-1 randomized clinical study [32] and in those who received first-line palbociclib plus letrozole or placebo plus letrozole $(2.0 \%$ and $2.3 \%$, respectively) in the PALOMA-2 randomized clinical study (data on file; Pfizer Inc, Full Clinical Study Report A5481008; 2016). The differences in CR rates observed between this present analysis and findings 


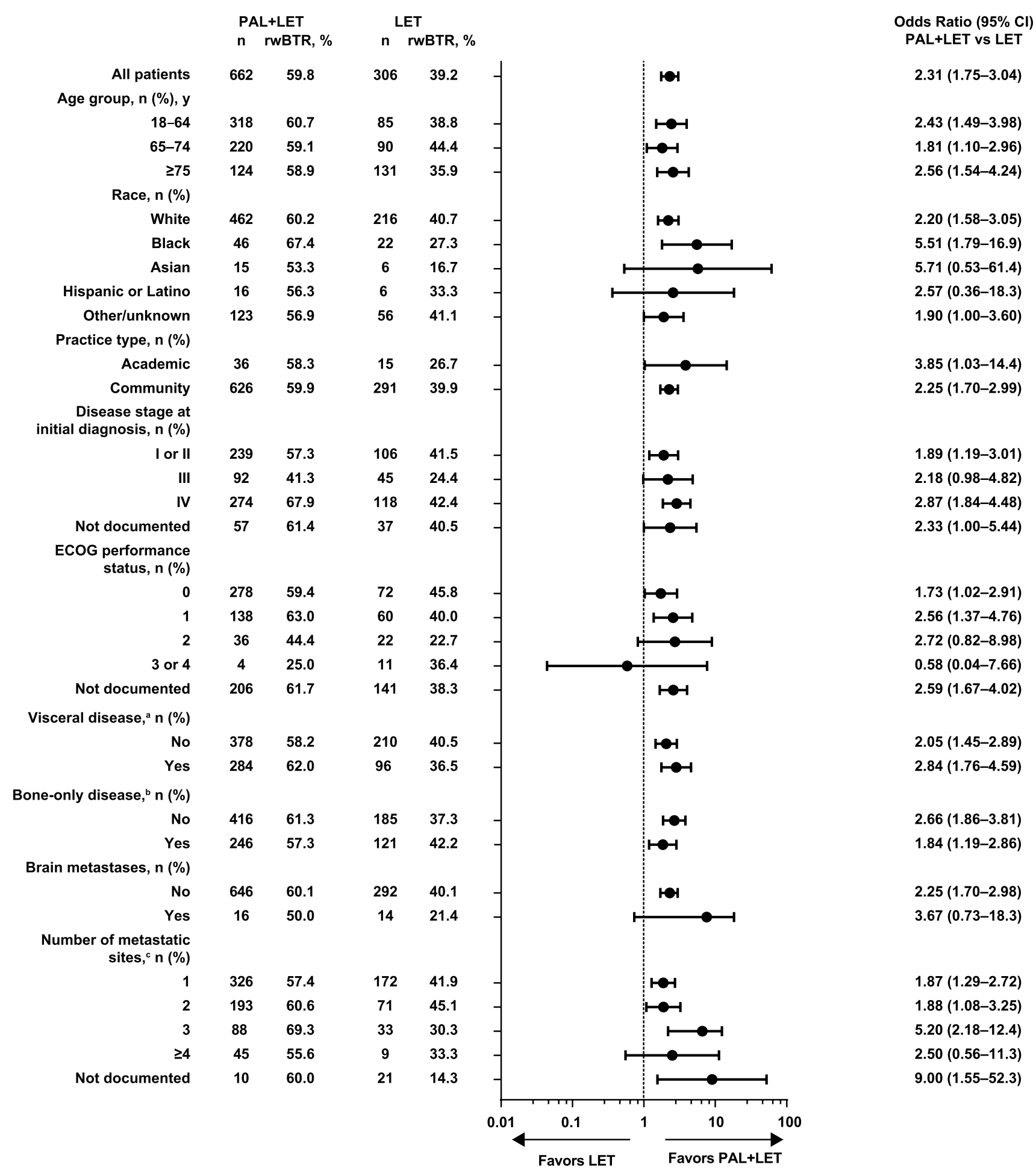

Fig. 2 Real-world best overall tumor response rates by subgroup. $E C O G$ Eastern Cooperative Oncology Group, LET letrozole, PAL palbociclib, $r w B T R$ real-world best tumor response ${ }^{a} V i s c e r a l$ disease was defined as metastatic disease in the lung and/or liver; patients could have had other sites of metastases. No visceral disease was defined as no lung or liver metastases. ${ }^{\mathrm{b}}$ Bone-only disease was defined as metastatic disease in only the bone. ${ }^{c}$ Multiple metastases at the same site were counted as one site (e.g., if a patient had three bone metastases in the spine, it was considered only one site) 

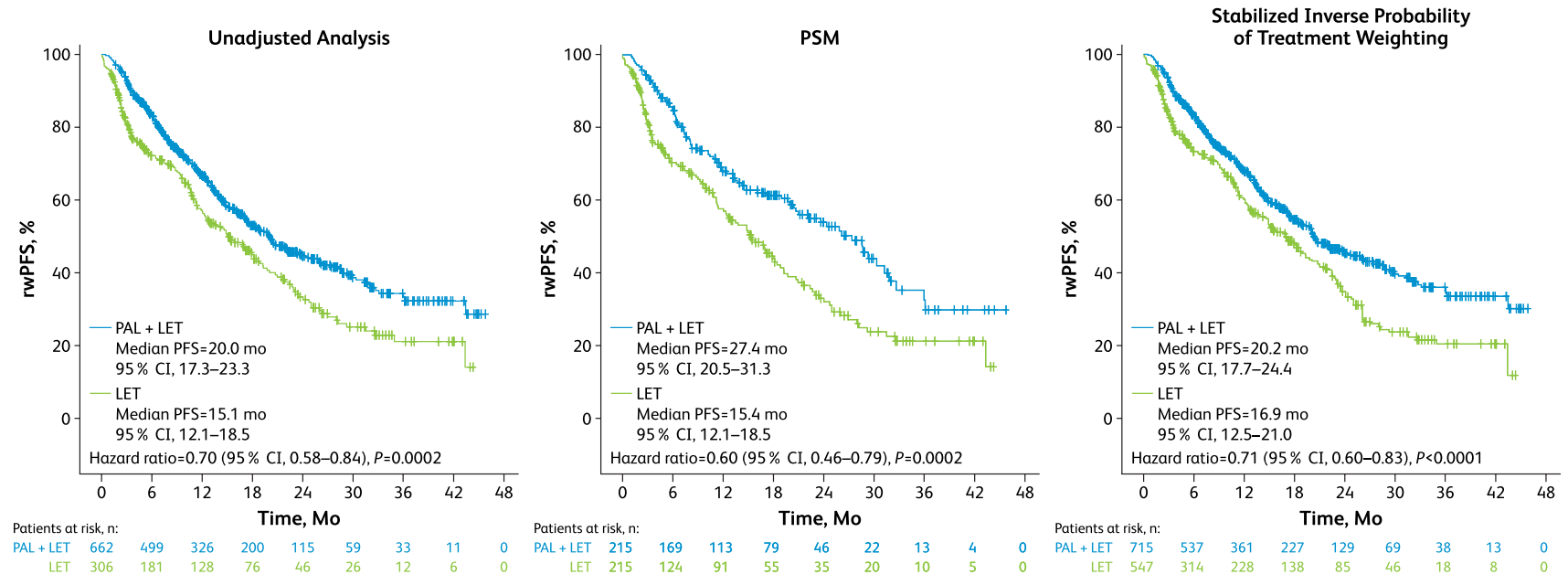

Fig. 3 Real-world progression-free survival among patients with one or more tumor response assessments. LET letrozole, $P A L$ palbociclib, $P S M$ propensity-score matching, rwPFS real-world progression-free survival
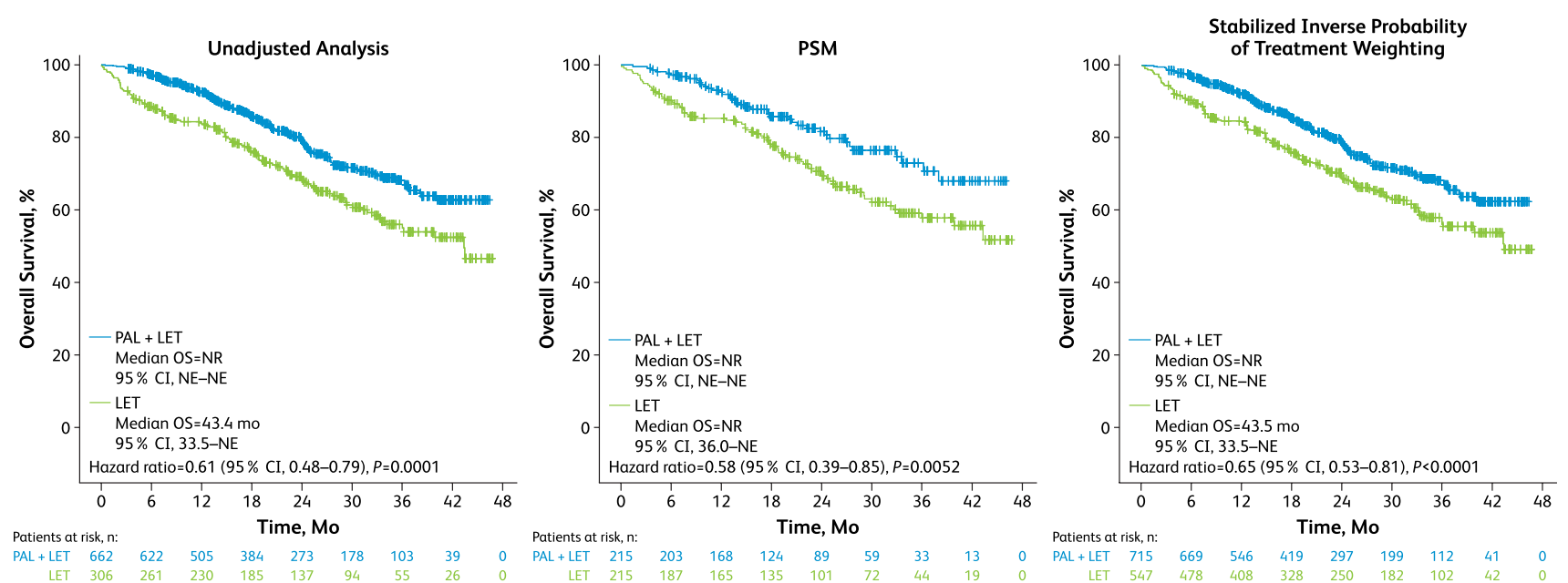

Fig. 4 Overall survival among patients with one or more tumor response assessments. $L E T$ letrozole, $N E$ not estimable, $N R$ not reached, $O S$ overall survival, $P A L$ palbociclib, $P S M$ propensity-score matching

from the randomized clinical studies can be attributed to the differences in tumor response assessment and criteria.

The present real-world data and previous results from palbociclib clinical studies were generally similar to findings shown in randomized clinical studies of other CDK4/6 inhibitors for the treatment of women with HR+/HER2$\mathrm{ABC}$ who had not received prior systemic therapy for advanced disease $[32,33]$. In the MONALEESA-2 study, the objective response rate among all patients was 47\% (95\% CI 38-57) in the ribociclib plus letrozole group and 34\% (95\% CI 25-42) in the placebo plus letrozole group [32]. In patients who had measurable disease, the objective response rate was 56\% (95\% CI 46-66) with ribociclib plus letrozole and $45 \%$ (95\% CI 34-55) with placebo plus letrozole [32]. Similarly, findings from the MONARCH 3 study reported an objective response rate of $48.2 \%$ (95\% CI 42.8-53.6) with abemaciclib plus a nonsteroidal AI and 34.5\% (95\% CI 27.3-41.8) with placebo plus a nonsteroidal AI [33]. In patients with measurable disease, the objective response rate was 59.2\% (95\% CI 53.3-65.1) in the abemaciclib group and $43.8 \%$ (95\% CI 35.3-52.4) in the placebo group [33]. Together, these studies highlight the consistency of tumor response data across all CDK4/6 inhibitors, and consistent with National Comprehensive Cancer Network guidelines [5], support CDK4/6 inhibitors in combination with ET as first-line therapy for HR+/HER2 - advanced/MBC.

This analysis also showed the benefit of palbociclib plus letrozole compared with letrozole alone in terms of median rwPFS and OS results. After PSM, median rwPFS was 27.4 months with palbociclib plus letrozole and 15.4 months 
with letrozole alone (hazard ratio, 0.60 (95\% CI 0.46-0.79), $P=0.0002)$; median OS was not reached in either the palbociclib plus letrozole or the letrozole alone group (hazard ratio, 0.58 (95\% CI 0.39-0.85), $P=0.0052$ ). These findings are consistent with a previous Flatiron Health real-world comparative effectiveness analysis; palbociclib plus letrozole was associated with longer median rwPFS and OS compared with letrozole alone (20.2 vs. 11.9 months and not reached vs. 43.1 months, respectively, both $P<0.0001$ ) [22]. These results also complement the results from the PALOMA-2 clinical study, which reported a median PFS of 27.6 months with palbociclib plus letrozole versus 14.5 months with placebo plus letrozole $(P<0.0001$; data cutoff: 31 May 2017) [7]. OS data from PALOMA-2 have not been reported yet.

The present findings should be interpreted in the context of the following limitations. First, this is a retrospective analysis of data from an EHR database and causal relationships to treatment cannot be determined. Second, similar to other EHR databases, the Flatiron Health database may have missing or erroneous data, including data that may not be missing at random [34]. Tumor response assessments were missing in a higher proportion of patients treated with letrozole alone compared with those treated with palbociclib plus letrozole. Third, tumor assessments in routine practice were not scheduled and were not confirmed by additional imaging. These data were limited by the clinician's interpretation and documentation of tumor response based on radiologic evidence for change in burden of disease, and tumor response assessment was not based on RECIST. Fourth, although PSM was used to balance patient characteristics and stabilized inverse probability of treatment weighting was used as a sensitivity analysis, other variables unavailable in the database, such as menopausal status, disease-free interval, and endocrine sensitivity could not be statistically controlled. Additionally, tolerability and adverse events data were not collected in the database and were not evaluated in this analysis. Moreover, these findings may not be generalizable to other patient populations.

Despite these limitations, the real-world tumor response findings and associated response benefit shown in patients treated with palbociclib plus letrozole are supported by the observed rwPFS and OS benefit. In addition, results from the stabilized inverse probability of treatment weighting sensitivity analysis were consistent with findings from the PSM analysis. Moreover, the real-world tumor response variable is less likely to be confounded by comorbid conditions, unobserved variables, or subsequent treatments that may affect PFS or OS. Although further real-world studies are needed, these findings add to the current body of evidence supporting the effectiveness of palbociclib combination therapy in the first-line setting, complementing the results from randomized clinical studies and other evidence of effectiveness endpoints. Real-world studies provide valuable insights into the benefits of palbociclib in a general oncology population of patients with $\mathrm{HR}+/ \mathrm{HER} 2-\mathrm{ABC} / \mathrm{MBC}$ treated in routine clinical practice, including those with demographic and disease characteristics that may not be represented in randomized clinical trials.

\section{Conclusion}

Patients with HR+/HER2- MBC treated with first-line palbociclib plus letrozole were associated with a significantly higher likelihood of tumor response than those treated with letrozole alone in this large cohort of general oncology practice patients in the USA. In addition, treatment with first-line palbociclib plus letrozole was associated with significant rwPFS and OS improvement compared with letrozole alone. These data complement the clinical benefit observed with palbociclib plus endocrine therapy in randomized clinical trials and support palbociclib plus letrozole as a standard of care for patients with HR+/HER2- MBC.

Supplementary Information The online version contains supplementary material available at https://doi.org/10.1007/s11523-021-00826-1.

Acknowledgements This study was sponsored by Pfizer Inc. Editorial support was provided by Anny Wu, PharmD, of ICON plc (North Wales, PA, USA), and was funded by Pfizer Inc.

\section{Declarations}

Funding Pfizer Inc (NCT04176354).

Conflict of interest Adam Brufsky has received consulting fees from Pfizer Inc, AstraZeneca, Sanofi, Eli Lilly, and Novartis. Xianchen Liu, Benjamin Li, and Lynn McRoy are employees of and own stock in Pfizer Inc. Rachel M. Layman's institution has received research funding from Pfizer Inc, Novartis, and Eli Lilly; and Dr. Layman has participated in advisory boards for Pfizer Inc, Eli Lilly, and Novartis.

Ethics approval This study is exempt from institutional review board approval because it is retrospective, non-interventional, and used anonymized data provided by Flatiron.

Consent to participate Not applicable.

Consent for publication Not applicable.

Availability of data and material Upon request, and subject to certain criteria, conditions, and exceptions (see https://www.pfizer.com/scien ce/clinical-trials/trial-data-and-results for more information), Pfizer will provide access to individual de-identified participant data from Pfizer-sponsored global interventional clinical studies conducted for medicines, vaccines, and medical devices (1) for indications that have been approved in the USA and/or EU or (2) in programs that have been terminated (i.e., development for all indications has been discontinued). Pfizer will also consider requests for the protocol, data dictionary, and statistical analysis plan. Data may be requested from Pfizer trials 24 
months after study completion. The de-identified participant data will be made available to researchers whose proposals meet the research criteria and other conditions, and for which an exception does not apply, via a secure portal. To gain access, data requestors must enter into a data access agreement with Pfizer. The data on the real-world cohort that support the findings of this study have been originated by Flatiron Health, Inc and were purchased by Pfizer from Flatiron Health Inc for the purpose of this research. Access to the de-identified data set is subject to a contractual agreement with Flatiron Health; for data access, interested researchers should contact DataAccess@ flatiron. com. A licensing agreement is legally required prior to sharing these data in order to safeguard sensitive patient information, and to ensure proper deidentification and compliance with applicable restrictions and requirements under HIPAA.

\section{Code availability Not applicable.}

Author contributions All authors contributed to the conception and design of the study, provided study materials, data interpretation and analysis, drafted the manuscript, and/or critically revised the manuscript for intellectual contribution. BL collected and assembled data. All authors approved the final version of the manuscript for submission.

Open Access This article is licensed under a Creative Commons Attribution-NonCommercial 4.0 International License, which permits any non-commercial use, sharing, adaptation, distribution and reproduction in any medium or format, as long as you give appropriate credit to the original author(s) and the source, provide a link to the Creative Commons licence, and indicate if changes were made. The images or other third party material in this article are included in the article's Creative Commons licence, unless indicated otherwise in a credit line to the material. If material is not included in the article's Creative Commons licence and your intended use is not permitted by statutory regulation or exceeds the permitted use, you will need to obtain permission directly from the copyright holder. To view a copy of this licence, visit http://creativecommons.org/licenses/by-nc/4.0/.

\section{References}

1. American Cancer Society. About Breast Cancer. 2019. https:// www.cancer.org/cancer/breast-cancer/about.html. Accessed 28 Sep 2020.

2. American Cancer Society. Breast Cancer Facts \& Figures 2019-2020. American Cancer Society, Inc. https://www.cancer.org/ content/dam/cancer-org/research/cancer-facts-and-statistics/ breast-cancer-facts-and-figures/breast-cancer-facts-and-figures2019-2020.pdf. Accessed 30 Mar 2021.

3. Westphal T, Gampenrieder SP, Rinnerthaler G, Greil R. Cure in metastatic breast cancer. Memo. 2018;11(3):172-9.

4. O'Shaughnessy J. Extending survival with chemotherapy in metastatic breast cancer. Oncologist. 2005;10(suppl 3):20-9.

5. National Comprehensive Cancer Network. NCCN Clinical Practice Guidelines in Oncology (NCCN Guidelines $\left.{ }^{\circledR}\right)$ Breast Cancer Version 5.2020. https://www2.tri-kobe.org/nccn/guideline/breast/ english/breast.pdf. Accessed 30 Mar 2021.

6. IBRANCE® (palbociclib). Full Prescribing Information, Pfizer Inc, New York, NY, 2019.

7. Rugo HS, Finn RS, Dieras V, Ettl J, Lipatov O, Joy AA, et al. Palbociclib plus letrozole as first-line therapy in estrogen receptor-positive/human epidermal growth factor receptor 2-negative advanced breast cancer with extended follow-up. Breast Cancer Res Treat. 2019;174(3):719-29.
8. Finn RS, Martin M, Rugo HS, Jones S, Im SA, Gelmon K, et al. Palbociclib and letrozole in advanced breast cancer. N Engl J Med. 2016;375(20):1925-36.

9. Batra A, Cheung WY. Role of real-world evidence in informing cancer care: lessons from colorectal cancer. Curr Oncol. 2019;26(suppl 1):S53-6.

10. Karim S, Xu Y, Kong S, Abdel-Rahman O, Quan ML, Cheung WY. Generalisability of common oncology clinical trial eligibility criteria in the real world. Clin Oncol (R Coll Radiol). 2019;31(9):e160-6.

11. Saad ED. Endpoints in advanced breast cancer: methodological aspects \& clinical implications. Indian J Med Res. 2011;134:413-8.

12. Roever L. Endpoints in clinical trials: advantages and limitations. Evid Based Med Pract. 2016;1(2):1-2.

13. US Department of Health and Human Services Food and Drug Administration Center for Drug Evaluation and Research (CDER), Center for Biologics Evaluation and Research (CBER). Guidance for Industry: Clinical Trial Endpoints for the Approval of Cancer Drugs and Biologics. Rockville, MD: US Department of Health and Human Services; 2018.

14. Huang Bartlett C, Mardekian J, Cotter MJ, Huang X, Zhang Z, Parrinello CM, et al. Concordance of real-world versus conventional progression-free survival from a phase 3 trial of endocrine therapy as first-line treatment for metastatic breast cancer. PLoS ONE. 2020;15(4):e0227256.

15. Xi J, Oza A, Thomas S, Ademuyiwa F, Weilbaecher K, Suresh R, et al. Retrospective analysis of treatment patterns and effectiveness of palbociclib and subsequent regimens in metastatic breast cancer. J Natl Compr Canc Netw. 2019;17(2):141-7.

16. Varella L, Eziokwu AS, Jia X, Kruse M, Moore HCF, Budd GT, et al. Real-world clinical outcomes and toxicity in metastatic breast cancer patients treated with palbociclib and endocrine therapy. Breast Cancer Res Treat. 2019;176(2):429-34.

17. Bui TBV, Burgers DM, Agterof MJ, van de Garde EM. Real-world effectiveness of palbociclib versus clinical trial results in patients with advanced/metastatic breast cancer that progressed on previous endocrine therapy. Breast Cancer (Auckl). 2019;13:1-6.

18. Torres M, Liu X, Mardekian J, McRoy L. Palbociclib plus an aromatase inhibitor as first-line therapy for metastatic breast cancer in US clinical practice: real-world progression-free survival analysis. Presented at: European Society for Medical Oncology (ESMO) Congress, September 27-October 1, 2019; Barcelona, Spain.

19. Waller J, Mitra D, Mycock K, Taylor-Stokes G, Milligan G, Zhan $\mathrm{L}$, et al. Real-world treatment patterns and clinical outcomes in patients receiving palbociclib for hormone receptor-positive, human epidermal growth factor receptor 2-negative advanced or metastatic breast cancer in Argentina: the IRIS study. J Glob Oncol. 2019;5:JGO1800239.

20. Taylor-Stokes G, Mitra D, Waller J, Gibson K, Milligan G, Iyer S. Treatment patterns and clinical outcomes among patients receiving palbociclib in combination with an aromatase inhibitor or fulvestrant for HR+/HER2-negative advanced/metastatic breast cancer in real-world settings in the US: results from the IRIS study. Breast. 2019;43:22-7.

21. Pizzuti L, Giordano A, Michelotti A, Mazzotta M, Natoli C, Gamucci T, et al. Palbociclib plus endocrine therapy in HER2 negative, hormonal receptor-positive, advanced breast cancer: a real-world experience. J Cell Physiol. 2019;234(6):7708-17.

22. DeMichele A, Cristofanilli M, Brufsky A, Liu X, Mardekian J, McRoy L, et al. Comparative effectiveness of first-line palbociclib plus letrozole versus letrozole alone for HR+/HER2- metastatic breast cancer in US real-world clinical practice. Breast Cancer Res. 2021;23(1):37.

23. Carter GC, Sheffield KM, Gossai A, Huang Y-J, Zhu YE, Bowman $\mathrm{L}$, et al. Initial real world treatment patterns and outcomes of 
abemaciclib for the treatment of HR+, HER2- metastatic breast cancer [abstract]. Presented at: San Antonio Breast Cancer Symposium, December 10-14, 2019; San Antonio, TX.

24. Harbeck N, Bartlett M, Spurden D, Hooper B, Zhan L, Rosta E, et al. CDK4/6 inhibitors in HR+/HER2- advanced/metastatic breast cancer: a systematic literature review of real-world evidence studies. Future Oncol. 2021;17:2107-22.

25. Curtis MD, Griffith SD, Tucker M, Taylor MD, Capra WB, Carrigan G, et al. Development and validation of a high-quality composite real-world mortality endpoint. Health Serv Res. 2018;53(6):4460-76.

26. Singal G, Miller PG, Agarwala V, Li G, Kaushik G, Backenroth $\mathrm{D}$, et al. Association of patient characteristics and tumor genomics with clinical outcomes among patients with nonsmall cell lung cancer using a clinicogenomic database. JAMA. 2019;321(14):1391-9.

27. Cowper DC, Kubal JD, Maynard C, Hynes DM. A primer and comparative review of major US mortality databases. Ann Epidemiol. 2002;12(7):462-8.

28. Austin PC. The use of propensity score methods with survival or time-to-event outcomes: reporting measures of effect similar to those used in randomized experiments. Stat Med. 2014;33(7):1242-58.
29. Weber WA. Assessing tumor response to therapy. J Nucl Med. 2009;50(suppl 1):1S-10S.

30. Geels P, Eisenhauer E, Bezjak A, Zee B, Day A. Palliative effect of chemotherapy: objective tumor response is associated with symptom improvement in patients with metastatic breast cancer. J Clin Oncol. 2000;18(12):2395-405.

31. Finn RS, Crown JP, Lang I, Boer K, Bondarenko IM, Kulyk SO, et al. The cyclin-dependent kinase 4/6 inhibitor palbociclib in combination with letrozole versus letrozole alone as firstline treatment of oestrogen receptor-positive, HER2-negative, advanced breast cancer (PALOMA-1/TRIO-18): a randomised phase 2 study. Lancet Oncol. 2015;16(1):25-35.

32. O'Shaughnessy J, Petrakova K, Sonke GS, Conte P, Arteaga CL, Cameron DA, et al. Ribociclib plus letrozole versus letrozole alone in patients with de novo HR+, HER2- advanced breast cancer in the randomized MONALEESA-2 trial. Breast Cancer Res Treat. 2018;168(1):127-34.

33. Goetz MP, Toi M, Campone M, Sohn J, Paluch-Shimon S, Huober $\mathrm{J}$, et al. MONARCH 3: abemaciclib as initial therapy for advanced breast cancer. J Clin Oncol. 2017;35(32):3638-46.

34. Wells BJ, Chagin KM, Nowacki AS, Kattan MW. Strategies for handling missing data in electronic health record derived data. eGEMS (Generating Evidence and Methods). 2013;1(3,Article 7):1-9. 